\begin{tabular}{|l|l|l||}
\hline \multicolumn{2}{|c|}{ PublisherInfo } \\
\hline \hline PublisherName & $:$ & BioMed Central \\
\hline \hline PublisherLocation & $:$ & London \\
\hline \hline PublisherImprintName & $:$ & BioMed Central \\
\hline \hline
\end{tabular}

\title{
Diving Medicine at Cyberounds
}

\begin{tabular}{|l|l|l||}
\hline \multicolumn{2}{|c|}{ ArticleInfo } \\
\hline \hline ArticleID & $:$ & 4243 \\
\hline \hline ArticleDOI & $:$ & $10.1186 /$ ccf-1999-webreport81 \\
\hline \hline ArticleCitationID & $:$ & webreport81 \\
\hline \hline ArticleSequenceNumber & $:$ & 31 \\
\hline \hline ArticleCategory & $:$ & Web report \\
\hline \hline ArticleFirstPage & $:$ & 1 \\
\hline \hline ArticleLastPage & $:$ & 2 \\
\hline \hline & $:$ & RegistrationDate : 1999-2-6 \\
ArticleHistory & $:$ & OnlineDate $1999-2-6$ \\
\hline \hline ArticleCopyright & $:$ & Current Science Ltd1999 \\
\hline \hline ArticleGrants & $:$ & \\
\hline \hline ArticleContext & $:$ & 1305433 \\
\hline \hline
\end{tabular}




\section{Overview}

The authors describe SCUBA diving physiology, minor and major complications ( $10 \%$ fatal) and barotrauma prevention and therapy.An exhaustive reference list is included.

\section{Content}

An up-to-date review about the complications of this increasingly popular leasure activity.

\section{Other comments}

25 May 1999

\section{Evaluation}

Registration at Cyberounds Homepage (free):http://www.cyberounds.com

\section{References}

1. Cyberounds. [http://www.cyberounds.com/conferences/pulmonary_medicine/]

This PDF file was created after publication. 